\title{
Dipterofauna em Cultura de Coqueiro Anão Verde Associada a Plantas Invasoras em Linhares, ES, Brasil
}

\author{
Emerson Fraga Comério ${ }^{\circledR}$ \& Vera Lucia Rodrigues Machado Benassi²
}

1. Universidade Estadual Paulista "Júlio de Mesquita Filho", Faculdade de Ciências Agrárias e Veterinárias de Jaboticabal, e-mail: emersoncomerio@hotmail.com (Autor para correspondência ${ }^{\varpi}$ ). 2. Instituto Capixaba de Pesquisa, Assistência Técnica e Extensão Rural, e-mail: vbenassi@incaper.es.gov.br

\section{EntomoBrasilis 6 (3): 217-226 (2013)}

Resumo. O objetivo deste estudo foi realizar um levantamento da fauna de Diptera (Insecta) em um cultivo de Cocos nucifera L. e analisar a influência de plantas invasoras sobre a sua ocorrência. As amostragens foram realizadas semanalmente durante o período de março de 2008 a fevereiro de 2009, utilizando armadilhas Moericke em uma área mantida roçada e outra com a presença de plantas invasoras. Foram obtidos 27.466 exemplares de dípteros, sendo que 73,6\% ocorreram na área com plantas invasoras e $26,4 \%$ na área mantida roçada. Dolichopodidae, Tachinidae e Drosophilidae representaram mais de $85 \%$ do total de exemplares coletados nas duas áreas.

Palavras-chave: Cocos nucifera; Controle Biológico Conservativo; Diptera; Inimigos Naturais.

\section{Dipterofauna in Coconut Palm Crop Associated with Invasive Plants in Linhares, ES, Brazil}

Abstract. This study aimed to survey the Diptera (Insecta) fauna in coconut palm crop and analyze the influence of invasive plants on this fauna. From February 2008 to March 2009 weekly samples were performed using Moericke traps in areas with and without invasive plants. A total of 27,466 exemplars were collected, and $73.6 \%$ occurred in area whit invasive plants and $26.4 \%$ in the area maintained mowing. Dolichopodidae, Tachinidae and Drosophilidae families represented more than $85 \%$ of the specimens collected in both areas.

Keywords: Cocos nucifera; Conservative Biological Control; Diptera; Natural Enemies.

$\mathbb{N}$ as últimas décadas, ocorreu uma intensificação do uso de insumos agrícolas para atender à demanda proveniente da adoção de Sistemas Convencionais de produção no Brasil, com o objetivo de maximizar a produção, não levando em consideração, a qualidade do produto final e a preservação ambiental (MARANGONi et al. 1995; CAMPANHOLA et al. 1997).

A preocupação crescente da sociedade com a preservação e a conservação ambiental tem resultado na busca pelo setor produtivo, de tecnologias para a implantação de sistemas de produção agrícola com enfoques ecológicos, rentáveis e socialmente justos. Esta busca de estratégias que reduzam a dependência da produção agrícola dos agroquímicos e consequentemente, dos impactos negativos ao meio ambiente, passa por plantios com novos arranjos vegetais que substituam os tradicionais monocultivos extensivos. Uma estratégia chave é restaurar a diversidade da paisagem agrícola dentro e no entorno da propriedade rural, onde a diversidade deve ser melhorada no tempo (Aguiar-MeneZes 2004).

Estudos recentes vêm mostrando que uma maior diversidade florística, por exemplo, causaria um impacto direto na comunidade de um determinado agroecossistema, por fornecer ambientes adequados a um maior número de espécies animais, aumentando consequentemente, a sua riqueza e diversidade (FAdini et al. 2001; Altieri et al. 2003; BARBOSA et al. 2003).

Nesse contexto, as plantas invasoras representam um importante componente da diversidade florística nos agroecossistemas. Em culturas de manejo convencional elas podem exercer estresse biótico direto sobre as culturas competindo por luz, umidade e alguns nutrientes, reduzindo a produção; além disso, são prejudiciais por serem hospedeiras de artrópodes pragas (AltiERI et al. 2003).

Por outro lado, no contexto do controle biológico conservativo, as plantas invasoras são consideradas benéficas, uma vez que fornecem recursos para os inimigos naturais, como alimentos suplementares (presas e hospedeiros alternativos), alimentos complementares (néctar, pólen, honeydew), modificação do microclima, refúgio após práticas agrícolas e hábitats para hibernação. Com a presença de plantas invasoras em cultivos agrícolas, há um incremento nas populações de inimigos naturais que por sua vez impõe maior mortalidade às pragas (DyER \& LANDIS 1997; Cottrell \& Yeargan 1998; Sengonca et al. 2002; Altieri et al. 2003; Reвeк et al. 2005). Todavia, para que haja o sucesso deste tipo de manejo faz-se necessário conhecer a composição da fauna de insetos e das plantas invasoras que ocorrem em determinado cultivo.

Os Diptera constituem uma das maiores ordens de insetos, com cerca de 152.000 espécies descritas (WIEGMANn et al. 2011), podendo ser encontrados nos mais variados ecossistemas, alimentando-se de diferentes substratos. Em ecossistemas naturais e agroecossistemas, muitas espécies desempenham significativo papel ecológico, podendo atuar como detritívoros, polinizadores e inimigos naturais de pragas agrícolas (BORROR \& DeLong 1988; Gallo et al. 2002).

Considerando a escassez de estudos que envolvem a comunidade de plantas invasoras e a sua fauna associada, este trabalho propôs estudar a influência da presença de plantas invasoras na dipterofauna em uma cultura de coqueiro anão verde. 


\section{MATERIAL E MÉTODOS}

O experimento foi conduzido em uma área de cinco hectares, cultivada com Cocos nucifera L. cv. Anã Verde, de nove anos de idade, plantados a espaçamentos de 7,5 m entre plantas em um arranjo triangular, localizada na Fazenda Experimental do Instituto Capixaba de Pesquisa, Assistência Técnica e Extensão Rural (INCAPER) $\left(19^{\circ} 25^{\prime} 6,959^{\prime \prime} \mathrm{S} / 4^{\circ} 4^{\prime} 30,026^{\prime \prime} \mathrm{W} /\right.$ altitude: $50 \mathrm{~m}$ ) em Linhares, ES (Figura 1). A classificação climática da região segundo Köeppen é Aw, tratando-se de clima tropical úmido, com inverno seco é verão quente e chuvoso (NóBrEGA et al. 2012).

As amostragens foram realizadas semanalmente no período de março de 2008 a fevereiro de 2009 (totalizando 52 coletas), com a utilização de doze armadilhas tipo Moericke, das quais seis instaladas em área com presença de plantas invasoras $\left(450 \mathrm{~m}^{2}\right)$ e igual número em área mantida roçada $\left(450 \mathrm{~m}^{2}\right)$; o restante da área cultivada foi mantido no limpo. As armadilhas foram confeccionadas com bandejas plásticas medindo $39 \mathrm{~cm}$ de comprimento x $29 \mathrm{~cm}$ de largura x $6 \mathrm{~cm}$ de altura, forradas com papel do tipo "Contact" de cor amarela, colocadas ao nível do solo, sob a copa das plantas e distanciadas a 22,5 $\mathrm{m}$ entre si; a distância entre as áreas correspondeu a $37,5 \mathrm{~m}$. Cada armadilha foi preenchida com 1,5 L de água, $15 \mathrm{~mL}$ de solução de formalina a $37 \%$ e gotas e detergente neutro (Figura 2 e 3). As armadilhas permaneceram ativas durante todo período de amostragem com retirada do material biológico semanal e, substituição da solução conservante.

O material coletado foi triado sob microscópio estereoscópio, onde os exemplares de dípteros foram separados dos demais grupos de insetos e fixados e conservados em álcool a 70\%. A identificação das famílias de Diptera foi realizada utilizando-se a chave taxonômica de Borror \& DeLong (1988). Os exemplares encontram-se preservados em via úmida na coleção entomológica do INCAPER de Linhares.

As amostragens de plantas invasoras foram realizadas quinzenalmente através de observações "in loco", as quais foram posteriormente identificadas de acordo com LoRENZI (2008).

A análise de correlação de Pearson foi utilizada para avaliar a influência de variáveis climáticas na abundância de dípteros nas duas áreas estudadas. Os dados de temperatura, umidade e precipitação média dos meses em que as armadilhas ficaram expostas foram fornecidos pelo Centro de Meteorologia e Recursos Hídricos do INCAPER (Tabela 1).

Os dados do total de dípteros coletados entre os tratamentos foram submetidos à análise de variância, sendo as médias comparadas pelo teste de Tukey a $5 \%$ de probabilidade. As análises foram feitas utilizando o programa estatístico BioEstat 5.o (Ayres et al. 2007).

Para a análise faunística foram utilizados os índices de Diversidade de Shannon-Wiener (H'), Equitabilidade (J), Frequência Relativa, Constância, Abundância e Dominância, de acordo com Silveira-Neto et al. (1976). Para os cálculos dos índices de Diversidade e Equitabilidade utilizou-se o programa DivEs - Diversidade de Espécies v2.o (RoDRigues 2005); para os demais índices utilizou-se o Microsoft Excel ${ }^{\circledR}$.

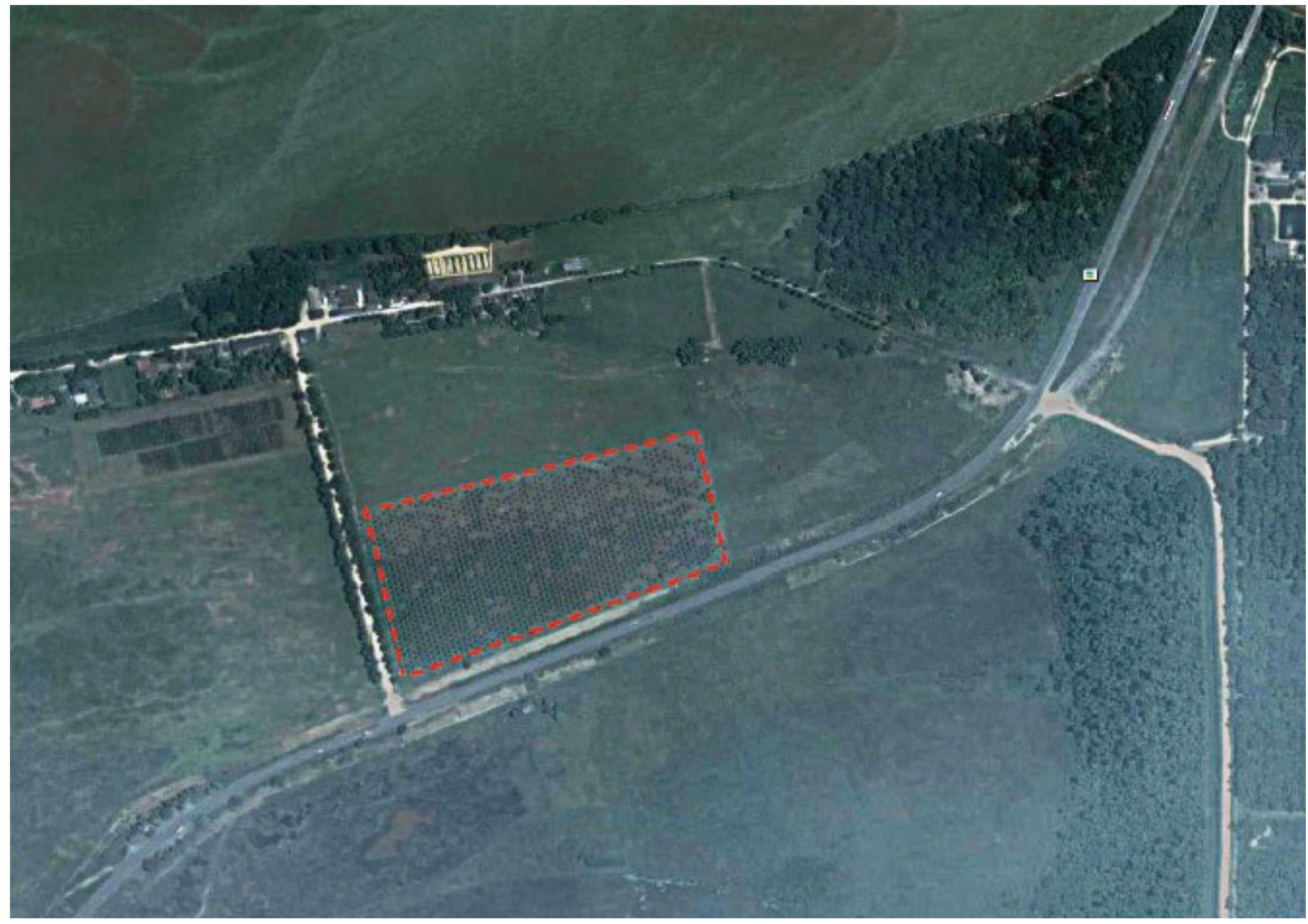

Figura 1. Vista aérea da Fazenda Experimental do Instituto Capixaba de Pesquisa, Assistência Técnica e Extensão Rural (INCAPER) (19² 25'6,959” S $/ 40^{\circ} 4$ '30,026" W / altitude: $50 \mathrm{~m}$ ) e, em destaque de vermelho a lavoura experimental de coqueiro anão verde, onde foi realizado o experimento, em Linhares, ES, Brasil. 

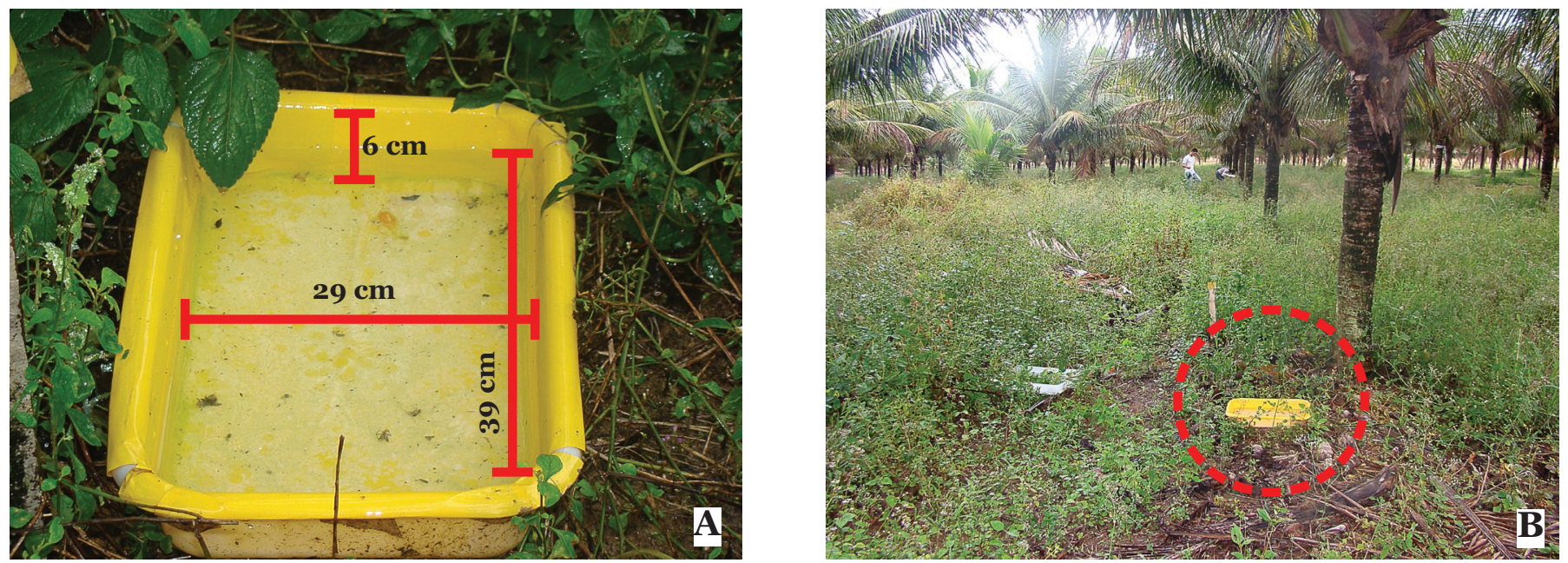

Figura 2. A) Armadilha Moericke utilizada na coleta dos insetos neste levantamento; B) Disposição da armadilha de Moericke na área de estudo, em destaque de vermelho.

Tabela 1. Dados de temperatura, umidade relativa e precipitação mensal, em cultura de coqueiro anão verde em Linhares, ES, entre março de 2008 e fevereiro de 2009

\begin{tabular}{cccc}
\hline Período de coleta & Temperatura média $\left({ }^{\circ} \mathbf{C}\right)$ & Precipitação $(\mathbf{m m})$ & Umidade relativa (\%) \\
\hline mar/08 & 25,9 & 98,4 & 80,6 \\
abr/08 & 26,1 & 101,4 & 79,5 \\
mai/o8 & 22,8 & 12,6 & 78,5 \\
\hline jun/o8 & 21,9 & 25,2 & 79,2 \\
jul/o8 & 20,8 & 30,8 & 77 \\
ago/o8 & 22,3 & 27 & 77,1 \\
set/o8 & 22,6 & 25,4 & 72,3 \\
out/08 & 24,6 & 86,2 & 75 \\
nov/o8 & 24 & 449,2 & 82,3 \\
dez/08 & 24,8 & 291,8 & 80,7 \\
jan/09 & 26 & 378 & 79,5 \\
fev/09 & 26,8 & 97,8 & 74,9 \\
\hline
\end{tabular}

\section{RESULTADOS E DISCUSSÃO}

Foram coletados 27.466 exemplares de dípteros, pertencentes a 23 famílias, dos quais $73,6 \%$ ocorreram na área com plantas invasoras e $26,4 \%$ na área mantida roçada (Tabela 2 e 3).

As médias do total de dípteros capturados entre os ambientes diferiram estatisticamente entre si (Tabela 4).

Embora o número de famílias tenha sido maior na área com plantas invasoras $(\mathrm{n}=23)$ do que na área mantida roçada $(\mathrm{n}=$ 19), o número de exemplares das famílias presentes somente na área com plantas (Sarcophagidae, Agromyzidae, Chloropidae e Cuterebridae) foi baixo. Um fator que pode ter contribuído para a semelhança entre o número de famílias nos dois tratamentos pode ser consequência da proximidade entre as áreas (37,5 m).

Ao longo dos meses de coleta a abundância de famílias e o número de exemplares coletados variaram, entretanto, notouse que as famílias que apresentaram os maiores valores de frequência relativa na área com plantas invasoras e na área mantida roçada respectivamente foram Dolichopodidae (70,0\% e $66,8 \%)$, Tachinidae (11,2\% e $13,0 \%)$ e Drosophilidae $(4,2 \%$ e $7,7 \%)$; as demais apresentaram frequências relativas inferiores a $3 \%$ em ambas as áreas.

A flutuação populacional dos dípteros ao longo do estudo mostrou que, os meses de maior captura foram agosto, setembro e outubro (final da estação seca e início da estação chuvosa na região) e, o mês de menor captura foi julho (estação seca), em ambas as áreas (Figura 4 e 5). Esses resultados corroboram com TorREs \& MADI-
RAvAZzi (2006) ao afirmarem que, geralmente observa-se que na estação chuvosa e quente, maior número de indivíduos é coletado, ao contrário da estação seca e fria. A ocorrência de chuvas favoreceu o florescimento das plantas invasoras, uma vez que a maioria das plantas observadas ocorreram floridas no período da estação chuvosa; isso consequentemente pode ter favorecido maior quantidade recursos alimentares e habitats, favorecendo na maior abundância de dípteros na estação chuvosa.

De acordo com Wolda (1980), o início das chuvas no final da estação seca funciona como gatilho para retorno da atividade dos insetos e, estudos desenvolvidos por Matthews \& Matthews (2010) mostraram que a atividade dos insetos diminui com baixas temperaturas, como nos meses de junho e julho, provocando queda do metabolismo, desaceleração do desenvolvimento dos insetos e consequentemente diminui seu forrageamento.

As análises de correlação de Pearson mostraram que somente foi observada correlação significativa e negativa entre abundância de dípteros e a variável umidade relativa em ambas às áreas ( $\mathrm{p}$ $\leq 0,05 \%)$; as demais variáveis, não foram observadas correlação significativa $(\mathrm{p} \geq 0,05 \%)$ :área com plantas invasoras [temperatura média $(\mathrm{r}=-0,0127)$, umidade relativa $(\mathrm{r}=-0,7143)$ e precipitação $(\mathrm{r}=0,4287)$ ] e, na área mantida roçada [temperatura média ( $\mathrm{r}$ $=0,1034)$, umidade relativa $(\mathrm{r}=-0,5976)$ e precipitação $(\mathrm{r}=$ $0,4287)]$.

É preciso salientar que, como este estudo foi realizado em um cultivo agrícola, não somente as variáveis climáticas influenciaram na captura dos indivíduos, mas também outros fatores como aplicação de defensivos agrícolas, roçagem, 
T Tabela 2. Número de dípteros coletados com armadilhas Moericke, em área com plantas invasoras, em cultura de coqueiro anão verde em Linhares, ES, entre março de 2008 e fevereiro de 2009.

\begin{tabular}{|c|c|c|c|c|c|c|c|c|c|c|c|c|c|}
\hline Família & $\frac{\infty}{\stackrel{\infty}{\varrho}}$ & $\frac{\infty}{0}$ & 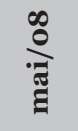 & 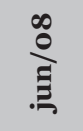 & 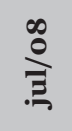 & 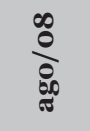 & $\underset{\substack{j \\
\vdots \\
\infty}}{\infty}$ & $\stackrel{\infty}{0}$ & $\stackrel{\infty}{\stackrel{0}{a}}$ & $\frac{\infty}{0}$ & 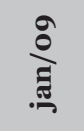 & $\frac{\partial}{d}$ & ฮี \\
\hline Dolichopodidae & 256 & 341 & 298 & 655 & 93 & 2947 & 2736 & 2765 & 804 & 530 & 1101 & 1621 & 14147 \\
\hline Tachinidae & 91 & 457 & 156 & 306 & 90 & 319 & 244 & 136 & 30 & 61 & 126 & 244 & 2260 \\
\hline Drosophilidae & 81 & 33 & 28 & 22 & o & 109 & 140 & 58 & 22 & 119 & 129 & 114 & 855 \\
\hline Micropezidae & 27 & 80 & 54 & 55 & 63 & 96 & 41 & 59 & 25 & 20 & 10 & 31 & 561 \\
\hline Mycetophilidae & 2 & 16 & o & o & o & o & 89 & 166 & 28 & 60 & 52 & 60 & 473 \\
\hline Pipunculidae & 5 & 5 & 16 & 12 & o & 37 & 48 & 104 & 11 & 32 & 70 & 46 & 386 \\
\hline Sciaridae & 30 & 49 & 5 & 9 & o & 11 & 12 & 32 & 14 & 51 & 33 & 47 & 293 \\
\hline Syrphidae & 11 & 64 & 46 & 68 & 7 & 15 & 19 & 21 & 4 & 11 & 13 & 23 & 302 \\
\hline Tephritidae & 11 & 28 & 31 & 61 & 16 & 13 & 5 & 13 & 5 & 30 & 3 & 15 & 230 \\
\hline Lonchaeidae & 24 & 5 & o & 11 & o & 10 & 45 & 63 & 15 & 7 & 19 & 53 & 252 \\
\hline Stratiomyidae & 6 & 15 & 18 & 5 & o & 9 & 15 & 28 & 9 & 15 & 3 & 12 & 135 \\
\hline Asilidae & o & o & 2 & 11 & 2 & 18 & 13 & 8 & 17 & 9 & 10 & 19 & 109 \\
\hline Muscidae & 2 & 1 & 1 & 2 & o & 2 & 7 & 4 & 4 & 7 & 17 & 13 & 60 \\
\hline Anisopodidae & o & 2 & o & o & o & 11 & 15 & 16 & 1 & 3 & o & o & 48 \\
\hline Otitidae & 3 & 4 & 1 & o & o & 5 & 3 & 2 & o & 6 & 2 & 8 & 34 \\
\hline Bombyliidae & o & 3 & 5 & 6 & 1 & 8 & 5 & o & o & o & 6 & o & 34 \\
\hline Calliphoridae & 5 & 3 & 5 & 3 & 3 & 1 & 1 & o & o & o & o & 1 & 22 \\
\hline Tabanidae & 3 & o & o & o & o & 6 & o & o & o & o & o & o & 9 \\
\hline Phoridae & 6 & o & o & o & o & o & o & o & o & o & o & o & 6 \\
\hline Sarcophagidae & o & o & o & o & o & o & o & o & o & 2 & o & 2 & 4 \\
\hline Chloropidae & o & o & o & o & o & o & 2 & o & o & o & o & o & 2 \\
\hline Cuterebridae & o & o & o & o & o & o & o & o & o & o & o & o & 1 \\
\hline Agromyzidae & $\mathrm{O}$ & o & o & o & o & 1 & o & o & o & o & o & o & 1 \\
\hline Total & 563 & 1106 & 666 & 1226 & 275 & 3618 & 3440 & 3475 & 989 & 963 & 1594 & 2309 & 20224 \\
\hline
\end{tabular}

Tabela 3. Número de dípteros coletados com armadilhas Moericke, em área mantida roçada, em cultura de coqueiro anão verde em Linhares, ES, entre março de 2008 e fevereiro de 2009.

\section{Família}

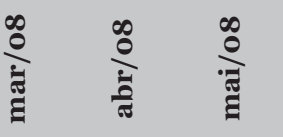

Dolichopodidae

Tachinidae

Drosophilidae

Micropezidae

Mycetophilidae

Pipunculidae

Sciaridae

Syrphidae

Tephritidae

Lonchaeidae

Stratiomyidae

Muscidae

Otitidae

Anisopodidae

Tabanidae

Bombyliidae

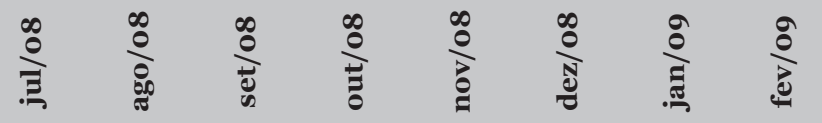

ฮี

$\begin{array}{ccccccccccccc}151 & 175 & 239 & 152 & 26 & 1169 & 512 & 1087 & 258 & 321 & 205 & 540 & 4835 \\ 45 & 179 & 67 & 70 & 37 & 114 & 50 & 45 & 13 & 29 & 106 & 185 & 940\end{array}$

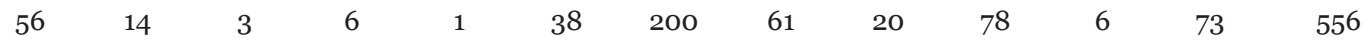

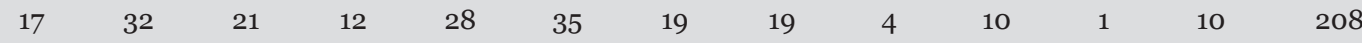

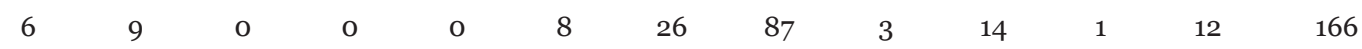

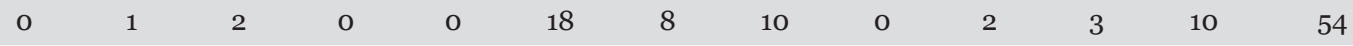

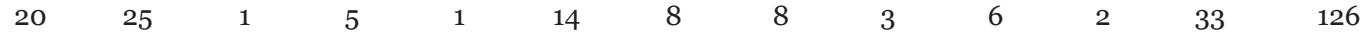

$\begin{array}{lllllllllllll}2 & 18 & 11 & 14 & 1 & 1 & 1 & 1 & 0 & 1 & 2 & 2 & 54\end{array}$

$\begin{array}{lllllllllllll}1 & 11 & 11 & 1 & 0 & 3 & 0 & 13 & 4 & 11 & 7 & 56 & 118\end{array}$

$\begin{array}{lllllllllllll}11 & 0 & 0 & 1 & 0 & 1 & 2 & 13 & 10 & 0 & 6 & 24 & 68\end{array}$

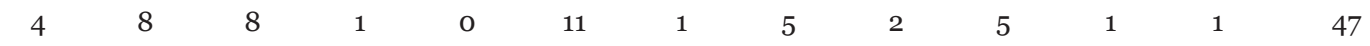

$\begin{array}{lllllllllllll}\mathrm{o} & \mathrm{o} & \mathrm{o} & 1 & \mathrm{o} & 2 & 4 & 8 & 2 & 2 & 6 & 10 & 35\end{array}$

$\begin{array}{lllllllllllll}0 & 1 & 0 & 0 & 0 & 0 & 3 & 5 & 0 & 0 & 0 & 0 & 9\end{array}$

\begin{tabular}{|c|c|c|c|c|}
\hline 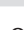 & 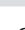 & & 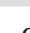 & \\
\hline
\end{tabular}

Continua... 
Tabela 3. Continuação...

\begin{tabular}{|c|c|c|c|c|c|c|c|c|c|c|c|c|c|}
\hline Família & 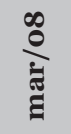 & $\begin{array}{l}\infty \\
0 \\
\frac{\hat{b}}{\sigma}\end{array}$ & 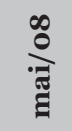 & $\stackrel{\infty}{\stackrel{0}{\ominus}}$ & $\stackrel{\infty}{\stackrel{0}{\ominus}}$ & 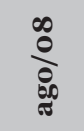 & $\frac{\infty}{0}$ & $\stackrel{\infty}{0}$ & $\frac{\infty}{0}$ & $\frac{\infty}{0}$ & 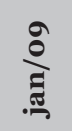 & $\frac{\varrho}{2}$ & 胥 \\
\hline Phoridae & 3 & o & o & o & o & o & o & o & o & o & o & o & 3 \\
\hline Asilidae & o & o & o & o & o & o & o & o & 2 & o & o & 1 & 3 \\
\hline Calliphoridae & 1 & o & 1 & o & o & o & o & o & o & o & o & o & 2 \\
\hline Total & 322 & 477 & 364 & 263 & 94 & 1415 & 835 & 1362 & 323 & 481 & 346 & 960 & 7242 \\
\hline
\end{tabular}

Tabela 4. Médias de dípteros coletados com armadilhas Moericke, em área com plantas invasoras e mantida roçada, em cultura de coqueiro anão verde em Linhares, ES, entre março de 2008 e fevereiro de 2009.

\begin{tabular}{lc}
\multicolumn{1}{c}{ Tratamento } & Média \\
\hline Área com plantas invasoras & $1.685,3 \mathrm{a}$ \\
Área mantida roçada & $603,5 \mathrm{~b}$ \\
CV (\%) & 79,8 \\
\hline
\end{tabular}

*Médias seguidas pela mestra letra na coluna, não diferem estatisticamente entre si pelo teste de Tukey, a $5 \%$ de probabilidade.

adubação/calagem e colheita, que foram realizados ao longo do experimento; esses fatores podem ter afetado a disponibilidade de recursos alimentares, habitas e refúgios para os dípteros capturados ao longo das semanas, além da presença de plantas invasoras, que fornecem maiores recursos e nichos ecológicos aos dípteros coletados. Brombal (2001) afirmou que práticas culturais influenciam na heterogeneidade do ambiente, bem como na disponibilidade de nutrientes das plantas e abrigo aos insetos fitófagos e seus inimigos naturais, e, ambientes mais heterogêneos atraem um maior número de artrópodes, de uma área menos diversificada para uma mais diversificada.

A elevada quantidade de Dolichopodidae capturada neste estudo pode ser creditada pelo fato desses insetos serem comumente capturados com uso de armadilhas de bandeja amarela, dispostas em lugares sombreados (Новаск et al. 1999), como na área estudada e, por apresentarem o hábito predatório, principalmente de coleópteros das famílias Scolytidae, Elateridae e Scarabaeidae (Sмiтн \& Empson 1955), e larvas de Diptera, Collembola, pulgões, tripes, ácaros e pequenas lagartas (UlRICH 2005), insetos observados durante as amostragens.

A família Tachinidae, a segunda mais abundante neste estudo, é a segunda em diversidade e importância ecológica como parasitoides (Toma \& Nihei 2006) e, para o coqueiro anão verde, várias espécies são citadas como importantes agentes de controle de pragas, a exemplo de Paratheresia menezesi Townsend e Billaea rhynchophorae (Blanchard) no controle das coleobrocas Rhynchophorus palmarum Linnaeus e Metamazius hemipterus (Linnaeus) (Coleoptera: Curculionidae); Winthemia analis (Macquart) parasitoide de lagartas de Brassolis sophorae Stichel (Lepidoptera: Nymphalidae) e, espécies de Plagiotachina Townsend e Sturmia Robineau-Desvoidy que parasitam lagartas de Automeris cinctistriga Felder (Lepidoptera: Saturniidae) (FERREIRa et al. 1997).

A presença de plantas invasoras na cultura pode ter favorecido um ambiente adequado para a manutenção de populações de taquinídeos, uma vez que, o maior número de exemplares foi observado nessa área. Adultos desses insetos necessitam diariamente de alimento açucarado fornecido por flores, conforme observado por TOPHAM \& BEARDSLEY JÚNIOR (1975) para Lixophaga sphenophori (Villeneuve), parasitoide da "broca-dacana-de-açúcar", que se alimenta de Euphorbia hirta L. Além disso, a heterogeneidade do ambiente oferecida pela vegetação invasora no cultivo amplia os locais de refúgio para esses insetos (Altieri et al 2003).
Os dípteros pertencentes à Drosophilidae alimentam-se de grande variedade de substratos orgânicos, como frutos em decomposição, flores, fungos, raízes, entre outros (TRIPLEHORN \& JoHnson 2005). A presença das plantas invasoras e de frutos de coqueiro em decomposição, que eram observados no solo, pode ter servido como local para alimentação e oviposição desses dípteros, corroborando com ARAúJo \& VALENTE (1981) que relataram que algumas espécies desses insetos alimentam-se de frutos de palmeiras em decomposição e flores de Asteraceae e Convolvulaceae, também observadas na área de estudo.

Embora as demais famílias tenham apresentado valores de frequência relativa mais baixos, algumas delas são importantes do ponto de vista do controle biológico, a exemplo dos representantes de Micropezidae que, de acordo com Merrit \& JAMES (1973), possuem hábito predatório; Pipunculidae, cujas larvas são endoparasitóides de várias famílias de HemipteraAuchenorryncha (HuQ 1984); Asilidae, que são predadoras generalistas e, Syrphidae que no estágio imaturo podem ser predadores de pulgões (GALlo et al. 2002); neste estudo, foram observados frequentemente durante as amostragens adultos de sirfídeos visitando flores e larvas se alimentando de pulgões que infestavam as plantas invasoras e, também larvas se alimentando de Cerataphis brasilisensis (Hempel) "pulgão-preto-docoqueiro" nos folíolos do coqueiro, o que é importante, pois este afídeo constitui-se praga para esta cultura (FERREIRA et al. 1997).

Mesmo nas famílias menos frequentes, sua maior abundância foi constatada na área com vegetação invasora, o que reforça a observação de que manutenção da diversidade vegetal favorece o incremento e a manutenção desses insetos, o que é importante para o cultivo para representantes das famílias de dípteros que agregam inimigos naturais. Os resultados das análises faunísticas encontram-se na Tabela 5 .

Observou-se que os índices de Diversidade e Equitabilidade não foram tão elevados e foram semelhantes em ambos os ambientes (área com plantas invasoras: $\mathrm{H}^{\prime}=0,544 ; \mathrm{J}=0,3995$ ) e (área mantida roçada: $\left.\mathrm{H}^{\prime}=0,5502 ; \mathrm{J}=0,4305\right)$. A baixa diversidade encontrada pode ser refletiva pela alta dominância exercida pelas famílias Dolichopodidae, Tachinidae e Drosophilidade, corroborando com Odum (2001), ao afirmar que a Equitabilidade J e H' se comportam inversamente à dominância, uma vez que os valores altos destes dois índices indicam uma baixa concentração de dominância. Além disso, essas famílias dominantes foram consideradas muito frequentes, muito abundantes e constantes, as demais foram frequentes ou pouco frequentes, acessórias ou raras, raras, dispersas, comuns ou abundantes. 

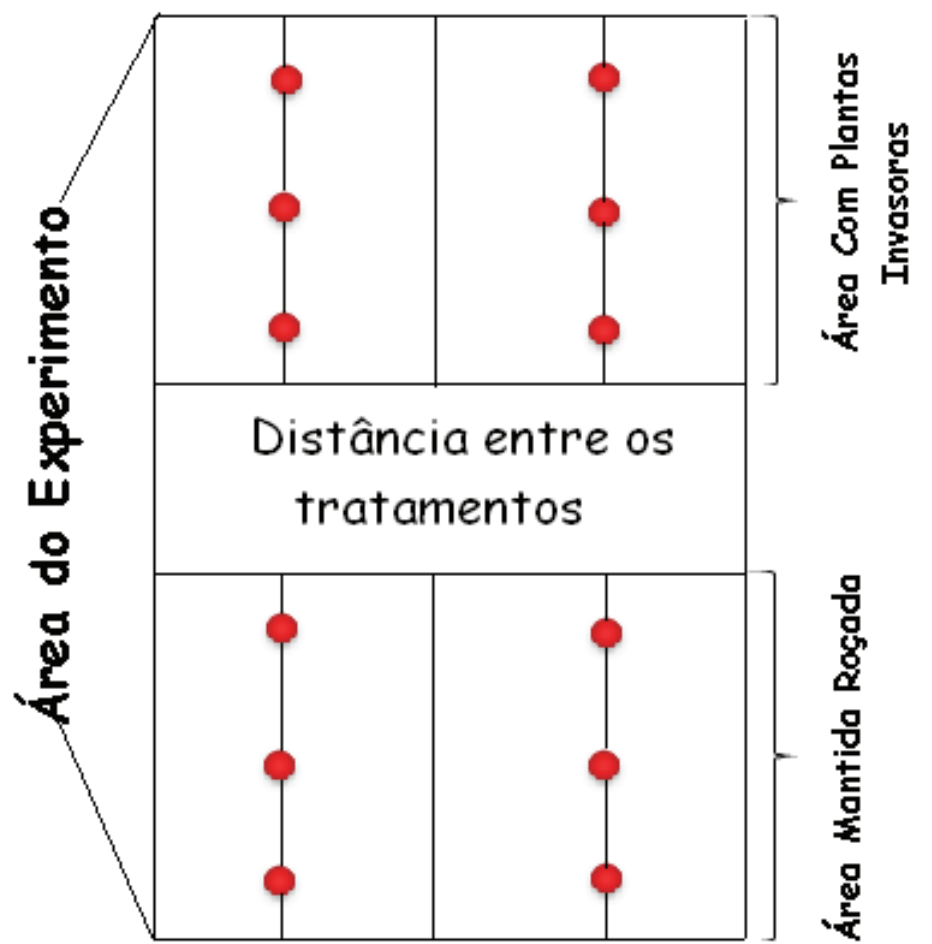

\section{ÁREATOTAL DO COQUEIRO}

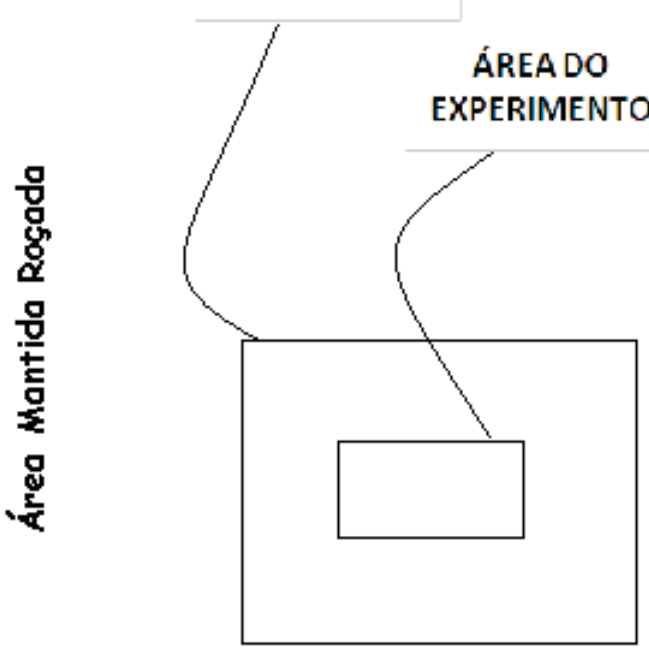

- Area total da lavoura de coqueiro: 5 hectares

- Área do experimento: $82,5 \mathrm{~m} \times 30 \mathrm{~m}$

- Espaçamento entre plantas na fileira: 7,5m

- Espaçamento entre fileiras: $7,5 \mathrm{~m}$

- Espaçamento entre armadilhas na fileira: $22,5 \mathrm{~m}$

- Área de cada tratamento: $15 \mathrm{~m}$ x 30m

- Distância entre os tratamentos: $37,5 \mathrm{~m}$

- : armadilha MOERICK.

Figura 3. Croqui da disposição geral do experimento na lavoura experimental de coqueiro anão verde em Linhares, ES, Brasil.

área com plantantas invasoras

$\square$ área mantida roçada

-temperatura média $\left({ }^{\circ} \mathrm{C}\right)$

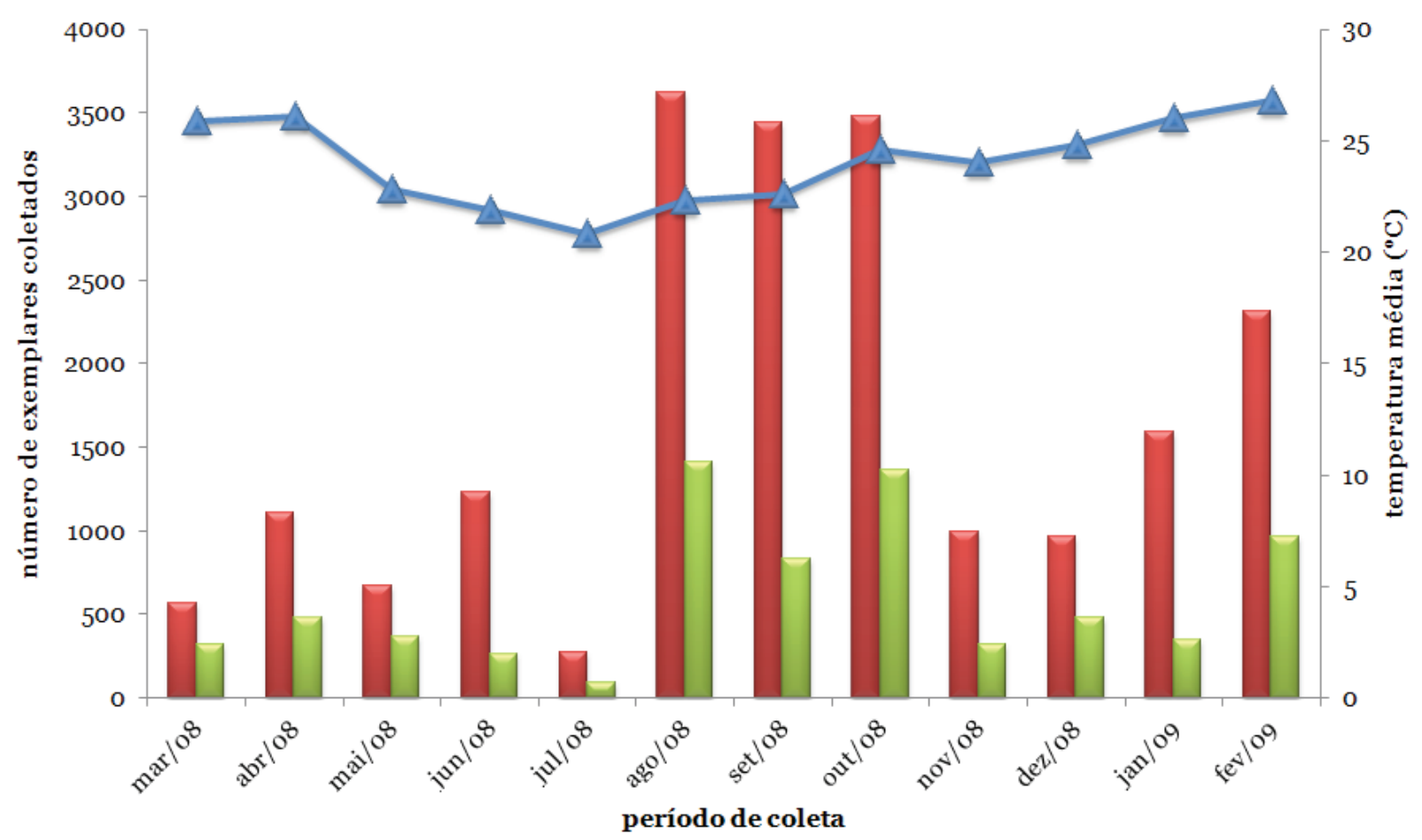

N

Figura 4. Influência da temperatura média $\left({ }^{\circ} \mathrm{C}\right)$ no número de dípteros coletados, em cultura de coqueiro anão verde em Linhares, ES, entre março de 2008 e fevereiro de 2009. 


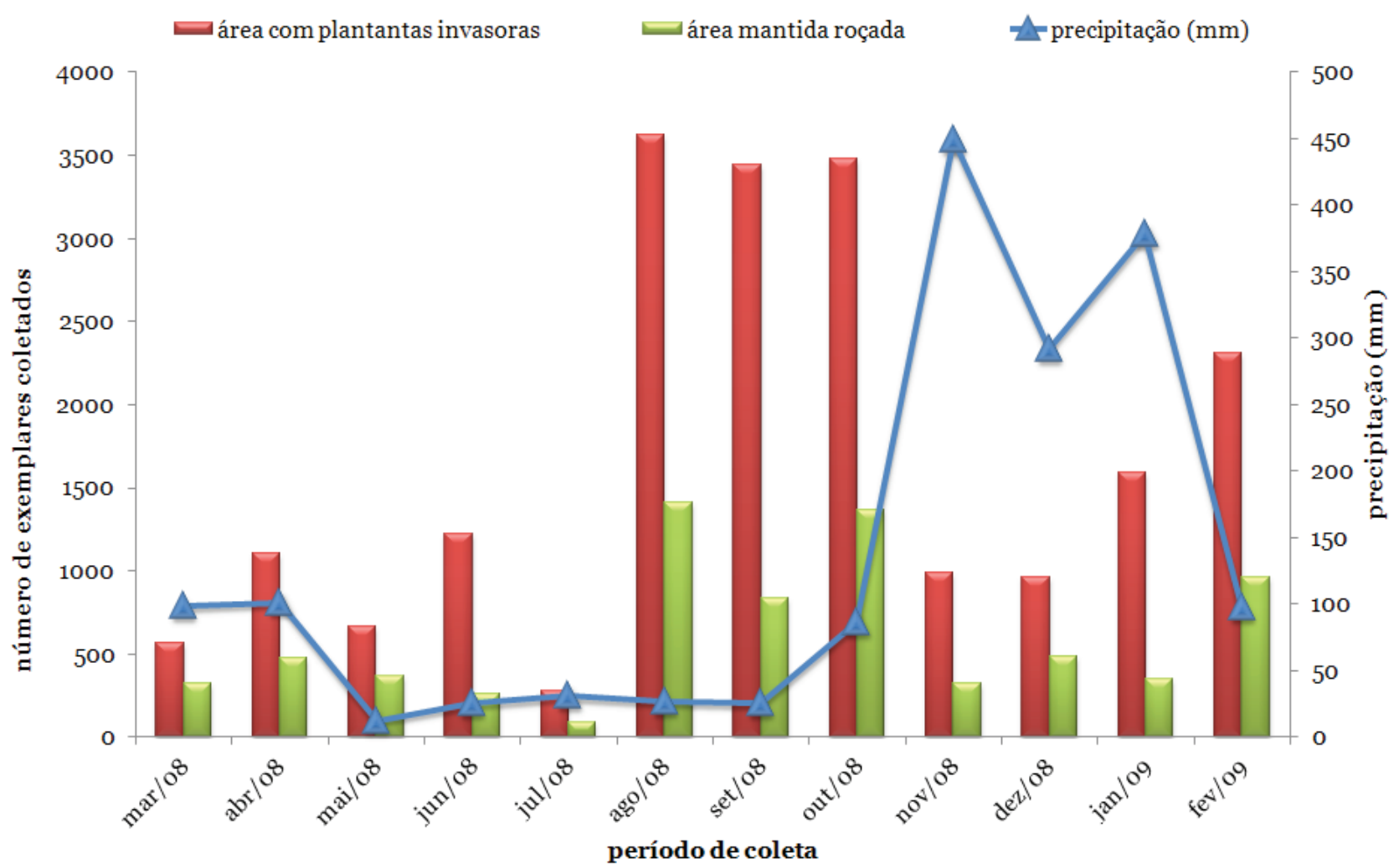

Figura 5. Influência da precipitação ( $\mathrm{mm}$ ) no número de dípteros coletados, em cultura de coqueiro anão verde em Linhares, ES, entre março de 2008 e fevereiro de 2009.

Tabela 5. Frequência, constância, abundância e dominância das famílias de Diptera (Insecta) coletados com armadilhas Moericke, em área com plantas invasoras e mantida roçada, em cultura de coqueiro anão verde em Linhares, ES, entre março de 2008 e fevereiro de 2009.

\begin{tabular}{|c|c|c|c|c|c|c|c|c|}
\hline \multirow[b]{2}{*}{ Família } & \multicolumn{4}{|c|}{ Área com plantas invasoras } & \multicolumn{4}{|c|}{ Área mantida roçada } \\
\hline & 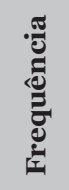 & 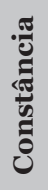 & 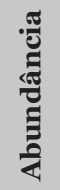 & 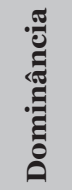 & & 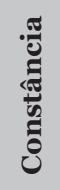 & 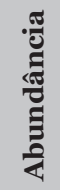 & 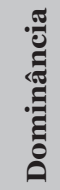 \\
\hline Dolichopodidae & MF & $\mathrm{W}$ & ma & $\mathrm{D}$ & MF & $\mathrm{W}$ & $\mathrm{ma}$ & $\mathrm{D}$ \\
\hline Tachinidae & MF & W & ma & $\mathrm{D}$ & MF & $\mathrm{W}$ & ma & $\mathrm{D}$ \\
\hline Drosophilidae & MF & $\mathrm{W}$ & ma & $\mathrm{D}$ & $\mathrm{MF}$ & $\mathrm{W}$ & $\mathrm{ma}$ & $\mathrm{D}$ \\
\hline Micropezidae & $\mathrm{F}$ & $\mathrm{W}$ & $\mathrm{c}$ & ND & $\mathrm{F}$ & $\mathrm{W}$ & $\mathrm{c}$ & ND \\
\hline Mycetophilidae & $\mathrm{F}$ & $\mathrm{W}$ & $\mathrm{c}$ & ND & $\mathrm{F}$ & $\mathrm{Y}$ & $\mathrm{c}$ & ND \\
\hline Pipunculidae & $\mathrm{F}$ & $\mathrm{W}$ & $\mathrm{c}$ & ND & $\mathrm{PF}$ & $\mathrm{Z}$ & d & ND \\
\hline Sciaridae & $\mathrm{F}$ & W & $\mathrm{c}$ & ND & $\mathrm{F}$ & $\mathrm{W}$ & $\mathrm{c}$ & ND \\
\hline Syrphidae & $\mathrm{F}$ & W & $\mathrm{c}$ & ND & $\mathrm{PF}$ & $\mathrm{Y}$ & d & ND \\
\hline Tephritidae & $\mathrm{F}$ & $\mathrm{W}$ & $\mathrm{c}$ & ND & $\mathrm{F}$ & $\mathrm{W}$ & $\mathrm{c}$ & ND \\
\hline Lonchaeidae & $\mathrm{F}$ & $\mathrm{W}$ & $\mathrm{c}$ & ND & $\mathrm{F}$ & $\mathrm{Y}$ & $\mathrm{c}$ & ND \\
\hline Stratiomyidae & $\mathrm{F}$ & $\mathrm{W}$ & $\mathrm{c}$ & ND & $\mathrm{F}$ & $\mathrm{Y}$ & $\mathrm{c}$ & ND \\
\hline Asilidae & $\mathrm{PF}$ & $\mathrm{Y}$ & d & ND & $\mathrm{PF}$ & $\mathrm{Z}$ & d & ND \\
\hline Muscidae & $\mathrm{PF}$ & $\mathrm{Y}$ & $\mathrm{d}$ & ND & $\mathrm{F}$ & $\mathrm{Y}$ & $\mathrm{c}$ & ND \\
\hline Anisopodidae & $\mathrm{PF}$ & $\mathrm{Y}$ & d & ND & $\mathrm{PF}$ & $\mathrm{Z}$ & d & ND \\
\hline Otitidae & $\mathrm{PF}$ & $\mathrm{Y}$ & d & ND & $\mathrm{PF}$ & $\mathrm{Z}$ & d & ND \\
\hline Bombyliidae & $\mathrm{PF}$ & $\mathrm{Y}$ & d & ND & $\mathrm{PF}$ & $\mathrm{Z}$ & d & ND \\
\hline Calliphoridae & $\mathrm{PF}$ & $\mathrm{Y}$ & d & ND & $\mathrm{PF}$ & $\mathrm{Z}$ & d & $\mathrm{ND}$ \\
\hline Tabanidae & $\mathrm{PF}$ & $\mathrm{Z}$ & d & ND & $\mathrm{PF}$ & $\mathrm{Z}$ & d & ND \\
\hline Phoridae & $\mathrm{PF}$ & $\mathrm{Z}$ & $\mathrm{d}$ & ND & $\mathrm{PF}$ & $\mathrm{Z}$ & $\mathrm{d}$ & ND \\
\hline Sarcophagidae & $\mathrm{PF}$ & $\mathrm{Z}$ & $\mathrm{d}$ & ND & - & - & - & - \\
\hline
\end{tabular}

Continua... 
Tabela 5. Continuação...

\begin{tabular}{|c|c|c|c|c|c|c|c|c|}
\hline \multirow[b]{2}{*}{ Família } & \multicolumn{4}{|c|}{ Área com plantas invasoras } & \multicolumn{4}{|c|}{ Área mantida roçada } \\
\hline & & 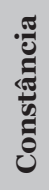 & 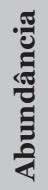 & 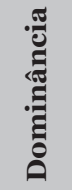 & & ن & 雚 & 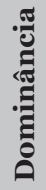 \\
\hline Chloropidae & $\mathrm{PF}$ & $\mathrm{Z}$ & $\mathrm{d}$ & ND & - & - & - & - \\
\hline Cuterebridae & $\mathrm{PF}$ & $\mathrm{Z}$ & $\mathrm{d}$ & ND & - & - & - & - \\
\hline Agromyzidae & $\mathrm{PF}$ & $\mathrm{Z}$ & $\mathrm{d}$ & ND & - & - & - & - \\
\hline
\end{tabular}

Frequência: $\mathrm{MF}=$ muito frequente; $\mathrm{F}=$ frequente; $\mathrm{PF}=$ pouco frequente.

Constância: $\mathrm{W}=$ constante; $\mathrm{Y}=$ acessória; $\mathrm{Z}=$ acidental.

Abundância: $\mathrm{ma}=$ muito abundante; $\mathrm{a}=$ abundante; $\mathrm{c}=$ comum; $\mathrm{d}=$ disperso.

Dominância: $\mathrm{D}=$ dominante; $\mathrm{ND}=$ não dominante.

De acordo com Silveira-Neto (1976) a presença de organismos dominantes, indica que estes provocam certo impacto na comunidade do ambiente, de forma que eles podem estar estabelecidos no habitat, reproduzindo-se e alimentando-se com êxito. Dessa maneira, este estudo fornece subsídios para que as espécies de taquinídeos e dolicopodídeos sejam mais detalhadamente investigadas do ponto de vista taxonômico e ecológico, visando conhecer seu papel no agroecossistema coqueiro anão verde estudado, uma vez que, estes grupos de dípteros podem atuar como inimigos naturais de pragas associadas ao coqueiro, como mencionado anteriormente e, dessa maneira este estudo demonstra a importância da aplicação de métodos de controle menos agressivos, que permitam a sua preservação.

As plantas invasoras observadas na área de estudo encontram-se listadas na Tabela 6.

As espécies da família Asteraceae foram observadas floridas durante quase todo o ano e em grande quantidade nas entrelinhas do cultivo, o que pode ter possibilitado elevado número de dípteros nas amostras; Brombal (2001) afirmou que asteráceas e outras dicotiledôneas são comuns em levantamentos florísticos em agroecossistemas e, se caracterizam por terem uma floração extensa ao longo do ano, apresentando uma arquitetura floral mais complexa, características essas que permitem fácil acesso ao néctar por grande número de dípteros e outros artrópodes.

Mexzón \& Chinchilla (1998) constataram que a presença de plantas invasoras nas entrelinhas do cultivo de dendezeiro Elaeis guineensis Jacq. na Costa Rica, favoreceu um incremento da fauna de dípteros predadores. Trabalhos de Altieri \& Schmidt (1987), Altieri \& Whitcomb (1980), Altieri et al. (2003) mostraram que, várias espécies de Asteraceae e Fabaceae têm desempenhado papel ecológico relevante por hospedarem um complexo de inimigos naturais, incluindo moscas predadoras e parasitoides, que atuam na supressão de populações de pragas.

Através do presente levantamento pode-se constatar que, de todas as famílias de dípteros coletadas, o maior número de

Tabela 6. Plantas invasoras registradas em cultura de coqueiro anão verde em Linhares, ES, entre de março de 2008 e fevereiro de 2009.

\begin{tabular}{|c|c|c|}
\hline Família/espécie & Nome vulgar & Ocorrência \\
\hline \multicolumn{3}{|l|}{ Asteraceae } \\
\hline Ageratum conyzoides L. & picão-roxo & mar a jul - set,out \\
\hline Bidens pilosa $\mathrm{L}$. & picão-preto & mar a jun - set, out, dez \\
\hline Emilia sanchifolia (L.) DC. & falsa-serralha & mar a ago \\
\hline Sonchus oleraceus L & serralha & mar a ago \\
\hline \multicolumn{3}{|l|}{ Amaranthaceae } \\
\hline Alternanthera tenella Colla & apaga-fogo & maio a out \\
\hline \multicolumn{3}{|l|}{ Commelinacea } \\
\hline Commelina benghalensis $\mathrm{L}$. & trapoeraba & mar, jun, jul, set, out \\
\hline \multicolumn{3}{|l|}{ Convolvulaceae } \\
\hline Ipomoea sp. & corda-de-viola & maio \\
\hline \multicolumn{3}{|l|}{ Euphorbiaceae } \\
\hline Euphorbia hirta L. & erva-de-santa-luzia & out \\
\hline \multicolumn{3}{|l|}{ Fabaceae } \\
\hline Cassia hirsuta L. & fedegoso-peludo & mar, jun, jul, set, out \\
\hline Desmodium barbatum (L.) & barbadinho & mar, jul \\
\hline Indigofera hirsuta L. & amores-do-campo & Jun \\
\hline \multicolumn{3}{|l|}{ Malvaceae } \\
\hline Sida sp. & guanxuma & fev, abr, jul, dez \\
\hline \multicolumn{3}{|l|}{ Rubiaceae } \\
\hline Borreria verticillata (L.) & vassourinha & maio \\
\hline Lantana camara $\mathrm{L}$. & erva-chumbinho & jun \\
\hline Stachytarphetta cayenensis (Rich.) M. & gervão & fev a abr, - jun a dez \\
\hline
\end{tabular}


exemplares foi representado por aquelas que incluem inimigos naturais. Embora este estudo não tenha sido conclusivo em relação ao impacto das plantas invasoras sobre a sua fauna, um número significativamente maior de famílias e de espécimes foi constatado na área com presença de plantas invasoras, o que sugerem que esses dípteros utilizam recursos provenientes dessa vegetação, favorecendo um incremento de suas populações no cultivo.

\section{REFERÊNCIAS}

Aguiar-Menezes, E.L., 2004. Diversidade Vegetal: uma estratégia para o manejo de pragas em sistemas sustentáveis de produção agrícola. Seropédica: Embrapa Agrobiologia. (Embrapa Agrobiologia. Documentos, 177). 68 p.

Altieri, M.A. \& L.L. Schmidt, 1987. Mixing cultivars of broccoli reduces cabbage aphid population. California Agriculture, Richmond, 41: 24-26.

Altieri, M.A., E.N. Silva \& C.I. Nicholls, 2003. O papel da biodiversidade no manejo integrado de pragas. Ribeirão Preto, Holos, 226p.

Altieri, M.A. \& W.H. Whitcomb, 1980. The potential use of weeds in the management in corn. Environmental Management, 4: 483-489.

Araújo, A.M. \& V.L.S. Valente, 1981. Observações sobre alguns lepidópteros e drosofilídeos do Parque do Truvo, RS. Ciência e Cultura, 33: 1485-1490.

Ayres, M., M. Ayres Jr, D.L. Ayres \& A. S. dos Santos, 2007. BioEstat 5.0 - aplicações estatísticas nas áreas das ciências biológicas e médicas. Belém, Sociedade Civil Mamirauá, 364 p.

Barbosa, F.R., Ferreira, R.G., Kill, L.H., Souza, E.A., Moreira, W.A., Alencar, J.A. \& F.N.P. Haji, 2003. Nível de dano, plantas invasoras hospedeiras, inimigos naturais e controle do psilídeo da goiabeira (Triozoida sp.) no submédio São Francisco. Revista Brasileira de Fruticultura, 25: 425-428.

Borror, D.J. \& D.M. DeLong, 1988. Introdução ao estudo dos insetos. São Paulo, Edgard Blucher, 653p.

Brombal, J.C., 2001. Estudo da fauna de Artrópodes Associada a plantas Invasoras em Agroecossistemas Orgânicos e Convencionais. 2001. Dissertação (Mestrado em Ecologia) - Programa de Pós-Graduação em Ecologia, Instituto de Biologia, Campinas. $141 \mathrm{p}$.

Campanhola, C., Luiz, A.J.B. \& A. Lucchiari-Júnior, 1997. O problema ambiental no Brasil: agricultura, p. 265-281. In: Romero, A.R., Reydon, B.P. \& M.L.A. Leonardi (Ed.). Economia do meio ambiente: teoria, políticas e gestão de espaços regionais. Campinas, UNICAMP. 384p.

Cottrell, T.E. \& K.V. Yeargan, 1998. Influence of a native weed, Acalypha ostryaefolia (Euphorbiaceae), on Coleomegilla maculata (Coleoptera: Coccinellidae) population density, predation, and cannibalism in sweet corn. Environmental Entomology, 27: 1375-1385

Dyer, L.E. \& D.A. Landis, 1997. Influence of noncrop habitats on the distribution of Eriborus terebrans (Hymenoptera, Ichneumonidae) in corn fields. Environmental Entomology, 26: 924-932.

Fadini, M.A., Regina, M.A., Fráguas, J.C. \& J.N.C. Louzada, 2001. Efeito da cobertura vegetal do solo sobre a abundância e diversidade de inimigos naturais de pragas em vinhedos. Revista Brasileira de Fruticultura, 23: 573-576.

Ferreira, J.M.S., R.N. Warnick \& L.A. Siqueira, 1997. A cultura do coqueiro no Brasil. Aracaju: EMBRAPA - CPTAC, 306p.

Gallo, D., O. Nakano, S. Silveira-Neto, R.P. Carvalho, G.C. Baptista, E. Berti-Filho, E., J.R.P. Parra, R.A. Zuchi, S.B. Alves, J.D. Vebdramim, L.C. Marchini, J.R.S. Lopes \& C. Omoto, 2002. Entomologia Agrícola. Piracicaba, Piracicaba, FEALQ - Biblioteca de Ciências Agrárias Luiz de Queiroz, 920p.

Hoback, W.W., T.M. Svatos, S.M. Spomer, \& L.G. Higley, 1999. Trap color and placement affects estimates of insect family- level abundance and diversity in a Nebraska salt marsh. Entomologia Experimentalis et Applicata, 91: 393-402.

Huq, S., 1984. Breeding methods for Pipunculidae (Diptera), endoparasites of leafhoppers. International Rice Research Newsletter, Manila, 9: 14-15.

Lorenzi, H., 2008. Plantas daninhas do Brasil. São Paulo, Instituto Plantarum, 672p.

Marangoni, B., M. Tagliavini \& D. Scudelari, 1995. La gestione de suelo I'rrigazione e la fertilizzazione del pesco, p. 273-294. In: Marangoni, B., Tagliavini, M. \& D. Scudelari, (Ed.). La Peschiciltura Veronese alla Soglia de 2000. Verona, Atti del convegno, 359p.

Matthews, R.W. \& J.R. Matthews, 2010. Insect behavior. New York, Spring Science. 519p.

Merritt, R.W. \& M. James, 1973. The Micropezidae of California (Diptera). Bulletin of the California Insect Survey, 14: 1-29.

Mexzón, R.G.M. \& C.M.I. Chinchilla, 1998. Plant species attractive to beneficial entomofauna in oil palm (Elaeis guineensis Jacq.) plantations in Costa Rica. Oil Palm Papers, 19: 1-22.

Nóbrega, N.E.F., J.G.F. da Silva, H.E.A. Ramos \& F.S. Pagung. 2012. Balanço hídrico climatológico e classificação climática de Thornthwaite e Köppen para o município de Linhares. ES. Disponível em <http://hidrometeorologia.incaper.es.gov.br/ arquivos pdf/publicacoes/CONIRD/1567 linhares.pdf $>$. Acesso em: 01.07.2013.

Odum, E.P., 2001. Fundamentos de Ecologia. Lisboa, Fund. Calouste Gulbenkian, 927p.

Rebek, E.J., C.S. Sadof \& L.M. Hanks, 2005. Manipulating the abundance of natural enemies in ornamental landscapes with floral resource plants. Biological Control, 33: 203-216.

Rodrigues, W.C., 2005. DivEs - Diversidade de espécies. Versão 2.o. Software e Guia do Usuário. Disponível em: $<$ http://www.ebras.bio.br>. Acesso 01.07.2013.

Sengonca, C., J. Kranz \& Blaeser, P. 2002. Attractiveness of three weed species to polyphagous predators and their influence on aphid populations in adjacent lettuce cultivations. Journal of Pesticide Science, 75: 161-165.

Silveira-Neto, S., O. Nakano, D. Barbin \& N.A. Villa-Nova, 1976. Manual de Ecologia dos insetos. São Paulo, Ceres, 419p.

Smith, G.V. \& D.W. Empson, 1955. Note on the coutship and predaceous behaviour of Paecilobothrus nobilitatus L. (Dip. Dolichopodidae). Animal Behaviour, 3: 32-34.

Toma, R. \& S.S. Nihei, 2006. Catálogo do material-tipo de Tachinidae (Diptera) depositado do Museu de Zoologia da Universidade de São Paulo. Revista Brasileira de Entomologia, 50: $240-256$.

Topham, M. \& J.W. Beardsaly Júnior, 1975. Influence of nectar source plants on the New Guinea sugar cane weevil parasite, Lixophaga sphenofori (Villeneuve). Proceedings of the Hawaiian Entomological Society, 22: 145-155.

Torres, F. R. \& L. Madi-Ravazzi, 2006. Seasonal variation in natural populations of Drosophila spp. (Diptera) in two woodlands in the State of São Paulo, Brazil. Ilheringia, Série Zoologia, 96: 437-444.

Triplehorn, C.A. \& N.F. Johnson, 2005. Borror's introduction to the study of insects. Washington, Brooks Cole, 864p.

Ulrich, H., 2005. Predation by adult Dolichopodidae (Diptera): a review of literature with an annotated prey-predator list. Studia Dipterologica, 11: 369-403.

Wiegmann, B.M., 2011. Episodic radiations in the fly tree of life. Proceedings of the National Academy of Sciences, 108: 56905695 .

Wolda, H., 1988. Insect seasonality: why? Annual Review of Ecology and Systematics, 19: 1-18.

Recebido em: 23/o4/2013

Aceito em: 09/07/2013

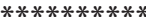


Como citar este artigo:

Comério, E.C. \& V.L.R.M. Benassi, 2013. Dipterofauna em Cultura de Coqueiro Anão Verde Associada a Plantas Invasoras em Linhares, ES, Brasil EntomoBrasilis, 6(3): 217-226.

Acessível em: http://www.periodico.ebras.bio.br/ojs/index.php/ebras/article/view/346. doi:10.12741/ebrasilis.v6i3.346
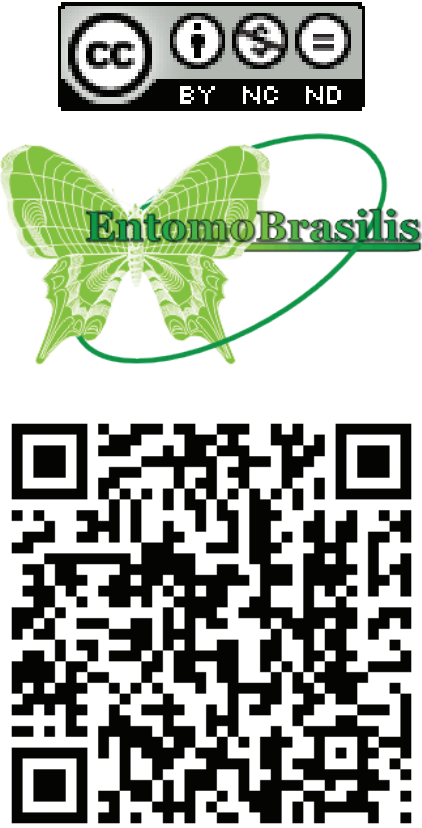